\title{
Optimization of Resource in the Industry of Construction
}

\author{
Francisco Javier Gutierrez Garcia, Pedro Pérez Díaz and Pedro Sanchez Luis \\ Department of Construction Engineering, University of La Laguna, Tenerife 38204, Spain
}

\begin{abstract}
Time and cost are two of the most important factors to consider in each construction project. In order to maximize performance, both the client and the contractor will work to optimize both the duration of the project and its cost. We show a model of linear entire mixed programming to solve the considered problem. The aim is to minimize the project total time, by means of the assignment of equipments of work to the different production lines of the activities to realizing. The fundamental beginning is to support the same production (rate of production in meters/day of the activity in view of the equipment of work) for the different equipments to achieve the maximum efficiency in each period of time. With the limited availability of resources, the work must be continuous and the period of time between operations and final must be kept. However, this paper also presents the bibliographical study on methodologies of the optimization of construction processes in response to the two objectives: time and cost. This will consider the use of meta-heuristic techniques, such as population based algorithms.
\end{abstract}

Key words: Optimization, construction process, heuristics, mathematical programming.

\section{Introduction}

The economic crisis that everybody is going through in Spain has targeted all sectors of industry, the construction industry being one of the most affected ones.

The fact that the construction industry is sensitive to the adverse financial conditions present in Spain, the limited financial resources and high competition in the market makes it necessary to perform project planning and control of works.

For years, builders have been limited to relying on experience and intuition, without regard to optimum needs of the resources used in the construction process. In small projects, the planning of resources can be developed by experience, but in large projects in which investments and, therefore, the risks are much higher, planning involves the optimization of processes that arise in the construction industry.

The principal aim of this paper is to make a bibliographical study on the methodologies raised in the associated document to support the planners and administrators in the capture of better decisions about

Corresponding author: Francisco Javier Gutierrez Garcia, engineer, research fields: physical and engineering. E-mail: fjguti@ull.es. time-cost in an efficient way.

Indexing the type of problems that we can meet, we have two types of problems of optimization: first, we find mono-objective problems of optimization, in which the development takes us to the optimization of the resources to reach the minimal cost of the project or the minimal term of execution in an independent way. In this type of work, it is not that the term of execution or the cost of the project is ignored, but depending on the variable in study the other one is taken as a constant. These types of problems are removed since, in most cases, the reduction of the term of execution takes into account an increase of the cost or, on the contrary, a reduction of the cost. Normally, it supposes an increase of the term of execution of any project. For this reason, the type of problems we really face are those in which a formulation is in use one multi-target, since the cost of a project and the term of execution are closely associated.

The second one of the principal aims of this work is to explore the analysis of the information corresponding to a practical case that one finds nowadays in the south of Tenerife, where only time studies are made.

Another contribution of this work is the mathematical formulation by means of a model of 
linear entire mixed programming to solve the problem. The comparative study is made from the time of execution of the work to the time provided by the mathematical formulation.

\section{Methodology}

\subsection{Bibliographical Review}

We show a bibliographical review of problems of optimization developed recently, mono-objective (Table 1) [1-7] and multi-objective (Table 2) [8-15]. These tables are arranged in six columns. In the first column, it shows the title of paper accessed, in the second, it shows the name of authors, the third shows the year of publishing, in the fourth column, it appears medium publishing and, finally, the fifth and sixth columns show the methodology and aim studied in the paper.

\subsection{Classification of Problems}

As shown in Tables 1 and 2, the classification of problems are as following:

- title of the paper or article;

- author(s) of the paper or article;
- year of publication of the paper or article;

- way of publication of the paper or article;

- mathematical proposed model on the paper or article (genetic algorithm, colony of ants and swarm of particles);

- type or aim of the work indexed on paper or article is reduction of project cost, reduced development time or reduction both, project cost and development time of the project.

\subsection{Selection of Article of Reference}

Resource-constrained scheduling is for the continuous repetitive projects with steels-based production units [16]. They show the complexity of the optimization with limited resources on fully linear works. This optimization is done regardless, at any time, the cost of funds and, therefore, the project cost.

\subsection{Comparison of the Real Time and the Optimized One}

We proposed a model of linear programming, with which we achieve a substantial reduction of project time and the project cost.

Table 1 Mono-objective references.

\begin{tabular}{|c|c|c|c|c|c|}
\hline Title & Author (s) & $\begin{array}{l}\text { Year of } \\
\text { publishing }\end{array}$ & Medium publishing & Methodology & Aim \\
\hline $\begin{array}{l}\text { Genetic algorithms for optimization } \\
\text { of resource allocation in large scale } \\
\text { construction project [1] }\end{array}$ & $\begin{array}{l}\text { Jianwen Huang, Xingxia } \\
\text { Wang, Rui Chen }\end{array}$ & 2010 & $\begin{array}{l}\text { Journal of } \\
\text { Computers }\end{array}$ & $\begin{array}{l}\text { Genetic } \\
\text { algorithms }\end{array}$ & $\begin{array}{l}\text { Minimize } \\
\text { project time }\end{array}$ \\
\hline $\begin{array}{l}\text { Optimizing construction planning } \\
\text { schedules by virtual prototyping } \\
\text { enabled resource analysis[2] }\end{array}$ & $\begin{array}{l}\text { Heng Li, Neo Chan, H.L. Guo, } \\
\text { Weisheng Lu, Martin } \\
\text { Skitmore } \\
\end{array}$ & 2009 & $\begin{array}{l}\text { QUT Digital } \\
\text { Repository }\end{array}$ & $\begin{array}{l}\text { Genetic } \\
\text { algorithms }\end{array}$ & $\begin{array}{l}\text { Minimize } \\
\text { project time }\end{array}$ \\
\hline $\begin{array}{l}\text { Simulation and optimization for } \\
\text { construction repetitive projects using } \\
\text { promodel and simrunner [3] }\end{array}$ & $\begin{array}{l}\text { Chachrist Srisuwanrat, Focio } \\
\text { G. Ioannou, Omer Tsimhoni }\end{array}$ & 2008 & $\begin{array}{l}\text { WSC (Winter } \\
\text { Simulation } \\
\text { Conference) } \\
\text { Phoenix, USA } \\
\end{array}$ & $\begin{array}{l}\text { Determinist } \\
\text { method }\end{array}$ & $\begin{array}{l}\text { Minimize } \\
\text { project time }\end{array}$ \\
\hline $\begin{array}{l}\text { Research on the unlimited resource } \\
\text { leveling optimization with PSO [4] }\end{array}$ & $\begin{array}{l}\text { Zhiyong Chen, Zhida Du, Hua } \\
\text { Zhou }\end{array}$ & 2007 & $\begin{array}{l}\text { China Civil } \\
\text { Engineering Journal }\end{array}$ & Particle swarm & $\begin{array}{l}\text { Minimize } \\
\text { project time }\end{array}$ \\
\hline $\begin{array}{l}\text { Construction project scheduling } \\
\text { problem with uncertain resource } \\
\text { constraints [5] }\end{array}$ & $\begin{array}{l}\text { Julin He, Zhong-Ping Wan, } \\
\text { Tang Guochun }\end{array}$ & 2005 & $\begin{array}{l}\text { Chinese Journal of } \\
\text { Engineering } \\
\text { Mathematics } \\
\end{array}$ & $\begin{array}{l}\text { Simple } \\
\text { algorithms }\end{array}$ & $\begin{array}{l}\text { Minimize } \\
\text { project time }\end{array}$ \\
\hline $\begin{array}{l}\text { Genetic algorithms in resource } \\
\text { optimization of construction project } \\
\text { [6] }\end{array}$ & $\begin{array}{l}\text { Zhang Lian-ying, Luo Gang, } \\
\text { Li Ning Lu }\end{array}$ & 2001 & $\begin{array}{l}\text { Journal of Tianjin } \\
\text { University }\end{array}$ & $\begin{array}{l}\text { Genetic } \\
\text { algorithms }\end{array}$ & $\begin{array}{l}\text { Minimize } \\
\text { project time }\end{array}$ \\
\hline $\begin{array}{l}\text { Resource leveling in construction by } \\
\text { genetic algorithm-based } \\
\text { optimization and its decision support } \\
\text { system application [7] }\end{array}$ & $\begin{array}{l}\text { Sou-Sen Leu, Chung-Huei } \\
\text { Yang, Jiun-Ching Huang }\end{array}$ & 2000 & $\begin{array}{l}\text { Journal of } \\
\text { Automation in } \\
\text { Construction }\end{array}$ & $\begin{array}{l}\text { Genetic } \\
\text { algorithms }\end{array}$ & $\begin{array}{l}\text { Minimize } \\
\text { project time }\end{array}$ \\
\hline
\end{tabular}


Table 2 Multi-objective references.

\begin{tabular}{|c|c|c|c|c|c|}
\hline Title & Author(s) & $\begin{array}{l}\text { Year of } \\
\text { publishing }\end{array}$ & Medium publishing & Methodology & Aim \\
\hline $\begin{array}{l}\text { Integrating efficient resource } \\
\text { optimization and linear schedule } \\
\text { analysis with singularity functions [8] }\end{array}$ & Lucko Gunnar & 2011 & $\begin{array}{l}\text { Journal of Construction } \\
\text { Engineering and } \\
\text { Management } \\
\end{array}$ & $\begin{array}{l}\text { Genetic } \\
\text { algorithms }\end{array}$ & $\begin{array}{l}\text { Minimize time } \\
\text { and project } \\
\text { cost }\end{array}$ \\
\hline $\begin{array}{l}\text { Optimization research: Enhancing the } \\
\text { robustness of large-scale } \\
\text { multi-objective optimization in } \\
\text { construction [9] }\end{array}$ & $\begin{array}{l}\text { Kandil Amr, } \\
\text { El-Rayes Khaled, } \\
\text { El-Anwar Omar }\end{array}$ & 2010 & $\begin{array}{l}\text { Journal of Construction } \\
\text { Engineering and } \\
\text { Management }\end{array}$ & $\begin{array}{l}\text { Genetic } \\
\text { algorithms }\end{array}$ & $\begin{array}{l}\text { Minimize time } \\
\text { and project } \\
\text { cost }\end{array}$ \\
\hline $\begin{array}{l}\text { Optimization study on resource } \\
\text { equilibrium with fixed time limit for a } \\
\text { project based on SPSO algorithm [10] }\end{array}$ & $\begin{array}{l}\text { Qingfu Li, Jixian } \\
\text { Guo, Mingxing Yi }\end{array}$ & 2008 & $\begin{array}{l}\text { International Symposium on } \\
\text { Intelligent Information } \\
\text { Technology Application } \\
\text { Workshops } \\
\text { Washington, DC, USA }\end{array}$ & $\begin{array}{l}\text { Genetic } \\
\text { algorithms }\end{array}$ & $\begin{array}{l}\text { Minimize time } \\
\text { and project } \\
\text { cost }\end{array}$ \\
\hline $\begin{array}{l}\text { Multiple resource constraint } \\
\text { time-cost-resource optimization using } \\
\text { genetic algorithm [11] }\end{array}$ & $\begin{array}{l}\text { Habib Fathi, Abbas } \\
\text { Afshar }\end{array}$ & 2008 & $\begin{array}{l}\text { First International } \\
\text { Conference on Construction } \\
\text { in Developing Countries } \\
\text { (ICCIDC-I)Pakistan }\end{array}$ & $\begin{array}{l}\text { Genetic } \\
\text { algorithms }\end{array}$ & $\begin{array}{l}\text { Minimize time } \\
\text { and project } \\
\text { cost }\end{array}$ \\
\hline $\begin{array}{l}\text { Multi-objective optimization for } \\
\text { large-scale highway construction } \\
\text { projects [12] }\end{array}$ & Amr A. Kandil & 2006 & $\begin{array}{l}\text { ProQuest, Dissertations \& } \\
\text { Theses }\end{array}$ & $\begin{array}{l}\text { Genetic } \\
\text { algorithms }\end{array}$ & $\begin{array}{l}\text { Minimize time } \\
\text { and project } \\
\text { cost }\end{array}$ \\
\hline $\begin{array}{l}\text { A hybrid mechanism for optimizing } \\
\text { construction simulation models [13] }\end{array}$ & $\begin{array}{l}\text { Taoming Cheng, } \\
\text { Chungwei Feng, } \\
\text { Yanliang Chen } \\
\end{array}$ & 2005 & $\begin{array}{l}\text { Journal of Automation in } \\
\text { Construction }\end{array}$ & $\begin{array}{l}\text { Genetic } \\
\text { algorithms }\end{array}$ & $\begin{array}{l}\text { Minimize time } \\
\text { and project } \\
\text { cost }\end{array}$ \\
\hline $\begin{array}{l}\text { Development and application of a } \\
\text { hybrid genetic algorithm for resource } \\
\text { optimization and management [14] }\end{array}$ & $\begin{array}{l}\text { O.O. Ugwu, J.H.M. } \\
\text { Tah }\end{array}$ & 2002 & $\begin{array}{l}\text { Engineering Construction } \\
\text { and Architectural } \\
\text { Management } \\
\text { WILEY (online library) }\end{array}$ & $\begin{array}{l}\text { Genetic } \\
\text { algorithms }\end{array}$ & $\begin{array}{l}\text { Minimize time } \\
\text { and project } \\
\text { cost }\end{array}$ \\
\hline $\begin{array}{l}\text { Optimizing resource utilization for } \\
\text { repetitive construction projects [15] }\end{array}$ & $\begin{array}{l}\text { Khaled El-Rayes, } \\
\text { Osama Moselhi }\end{array}$ & 2001 & $\begin{array}{l}\text { Journal of Management in } \\
\text { Engineering (ASCE) }\end{array}$ & $\begin{array}{l}\text { Genetic } \\
\text { algorithms }\end{array}$ & $\begin{array}{l}\text { Minimize time } \\
\text { and project } \\
\text { cost }\end{array}$ \\
\hline
\end{tabular}

\section{Problem of Reference}

The mathematical model is proposed by the teachers/aces [16].

It includes the following restrictions:

- Limited availability of multiple resources;

- The work must be constant and must not have dead times;

- The delivery time between operations and end must be kept.

\section{Case Study}

IDAM (installation of desalination seawater) of Granadilla de Abona is developed by the Consortium Ferrovial-Agromán Corporation and Cadagua.

\subsection{Activities}

Activity A: ML (linear meter) of placement of prefabricated girder, cover water tanks running with precast concrete prestressed material.

Activity B: ML of placement of alveolar plate.

\subsection{Available Resources and Combinations}

We show the available resources and combinations in Table 3.

\subsection{Choice}

In Table 4, we show our selection between different combinations.

In this table, it showed: activity, sub-activity and our selection as a function of available resources and daily production combining different choices.

The symbol A in table 3 (A) reference is coefficient relating on linear meters and square meters and the value is 4 per linear meter have 4 square meters.

\subsection{Plans of Work}

In Table 5, we show 13 different plans works what 
Table 3 Available resources and combinations.

\begin{tabular}{llllll}
\hline \multirow{2}{*}{ Activity } & \multirow{2}{*}{ Sub-activity } & \multicolumn{3}{c}{ Available resources } & Production \\
\cline { 3 - 6 } & & Autoderrick crane (ud) & Lift (ud) & Workers (ud) & M1 (linear meters) \\
\hline \multirow{4}{*}{ A } & 2.00 & 2.00 & 6.00 & Day ud \\
& & 1.00 & 2.00 & 3.00 & 95.85 days \\
& A2 & 1.00 & 2.00 & 2.00 & 43.20 \\
& A3 & 2.00 & 2.00 & 3.00 & 95.85 \\
& A4 & 1.00 & 2.00 & 6.00 & 95.85 \\
& A5 & 2.00 & 2.00 & 2.00 & $43.20 \mathrm{~A}$ \\
\hline \multirow{3}{*}{ B } & 1.00 & 2.00 & 3.00 & $48.62 \mathrm{~A}$ \\
& B1 & 1.00 & 0.00 & 3.00 & $48.62 \mathrm{~A}$ \\
& B2 & 1.00 & 1.00 & 2.00 & $27.13 \mathrm{~A}$ \\
& B3 & 2.00 & 0.00 & 6.00 & $95.85 \mathrm{~A}$ \\
& B4 & 2.00 & 0.00 & 5.00 & $75.76 \mathrm{~A}$ \\
& B5 & 2.00 & 2.00 & 5.00 & $75.76 \mathrm{~A}$ \\
\hline
\end{tabular}

Table 4 Choice.

\begin{tabular}{llllll}
\hline \multirow{2}{*}{ Activity } & Sub-activity & Available resources & Available resources & Available resources & Production \\
\cline { 3 - 6 } & & Crane & Lift & Workers & ML/day \\
\cline { 2 - 5 } & A1 & 1.00 & 2.00 & 6.00 & 95.85 \\
\hline A & B4 & 2.00 & 2.00 & 3.00 & $95.85 \mathrm{~A}$ \\
\hline
\end{tabular}

Total activity $\mathrm{A}=1,349.68 \mathrm{ML}$ placement of prefabricated girder; Total activity $\mathrm{B}=1,349.68 \mathrm{~A}$ ML placement of alveolar plate.

Table 5 Plans of work.

\begin{tabular}{lllllllll}
\hline \multirow{2}{*}{ Number plan } & \multicolumn{7}{c}{ Index of production } \\
\cline { 2 - 8 } & Week 1 & Week 2 & Week 3 & Week 4 & Week 5 & Week 6 & Week 7 & Week 8 \\
\hline 1 & $194.48 \mathrm{~A}$ & $194.48 \mathrm{~A}$ & $194.48 \mathrm{~A}$ & $194.48 \mathrm{~A}$ & $194.48 \mathrm{~A}$ & $194.48 \mathrm{~A}$ & $194.48 \mathrm{~A}$ & $8.13 \mathrm{~A}$ \\
3 & $200.93 \mathrm{~A}$ & $200.93 \mathrm{~A}$ & $200.93 \mathrm{~A}$ & $200.93 \mathrm{~A}$ & $200.93 \mathrm{~A}$ & $200.93 \mathrm{~A}$ & $163.95 \mathrm{~A}$ & \\
4 & $205.61 \mathrm{~A}$ & $205.61 \mathrm{~A}$ & $205.61 \mathrm{~A}$ & $205.61 \mathrm{~A}$ & $205.61 \mathrm{~A}$ & $205.61 \mathrm{~A}$ & $135.94 \mathrm{~A}$ & \\
5 & $210.28 \mathrm{~A}$ & $210.28 \mathrm{~A}$ & $210.28 \mathrm{~A}$ & $210.28 \mathrm{~A}$ & $210.28 \mathrm{~A}$ & $210.28 \mathrm{~A}$ & $107.87 \mathrm{~A}$ \\
6 & $214.95 \mathrm{~A}$ & $214.95 \mathrm{~A}$ & $214.95 \mathrm{~A}$ & $214.95 \mathrm{~A}$ & $214.95 \mathrm{~A}$ & $214.95 \mathrm{~A}$ & $79.84 \mathrm{~A}$ & \\
7 & $219.63 \mathrm{~A}$ & $219.63 \mathrm{~A}$ & $219.63 \mathrm{~A}$ & $219.63 \mathrm{~A}$ & $219.63 \mathrm{~A}$ & $219.63 \mathrm{~A}$ & $51.80 \mathrm{~A}$ \\
8 & $224.30 \mathrm{~A}$ & $224.30 \mathrm{~A}$ & $224.30 \mathrm{~A}$ & $224.30 \mathrm{~A}$ & $224.30 \mathrm{~A}$ & $224.30 \mathrm{~A}$ & $23.76 \mathrm{~A}$ \\
9 & $228.97 \mathrm{~A}$ & $228.97 \mathrm{~A}$ & $228.97 \mathrm{~A}$ & $228.97 \mathrm{~A}$ & $228.97 \mathrm{~A}$ & $224.70 \mathrm{~A}$ & & \\
10 & $233.64 \mathrm{~A}$ & $233.64 \mathrm{~A}$ & $233.64 \mathrm{~A}$ & $233.64 \mathrm{~A}$ & $233.64 \mathrm{~A}$ & $201.33 \mathrm{~A}$ & & \\
11 & $238.32 \mathrm{~A}$ & $238.32 \mathrm{~A}$ & $238.32 \mathrm{~A}$ & $238.32 \mathrm{~A}$ & $238.32 \mathrm{~A}$ & $177.97 \mathrm{~A}$ & & \\
12 & $242.99 \mathrm{~A}$ & $242.99 \mathrm{~A}$ & $242.99 \mathrm{~A}$ & $242.99 \mathrm{~A}$ & $242.99 \mathrm{~A}$ & $136.94 \mathrm{~A}$ & & \\
13 & $378.80 \mathrm{~A}$ & $378.80 \mathrm{~A}$ & $378.80 \mathrm{~A}$ & $215.48 \mathrm{~A}$ & & & & \\
\hline
\end{tabular}

Table 6 Restrictions of work.

\begin{tabular}{lll}
\hline \multirow{2}{*}{ ID } & \multicolumn{2}{c}{ Distances } \\
\cline { 2 - 3 } & LT (days) & LD (ML) \\
\hline ML girder & 1.00 & 95.85 \\
ML plate & & \\
\hline
\end{tabular}




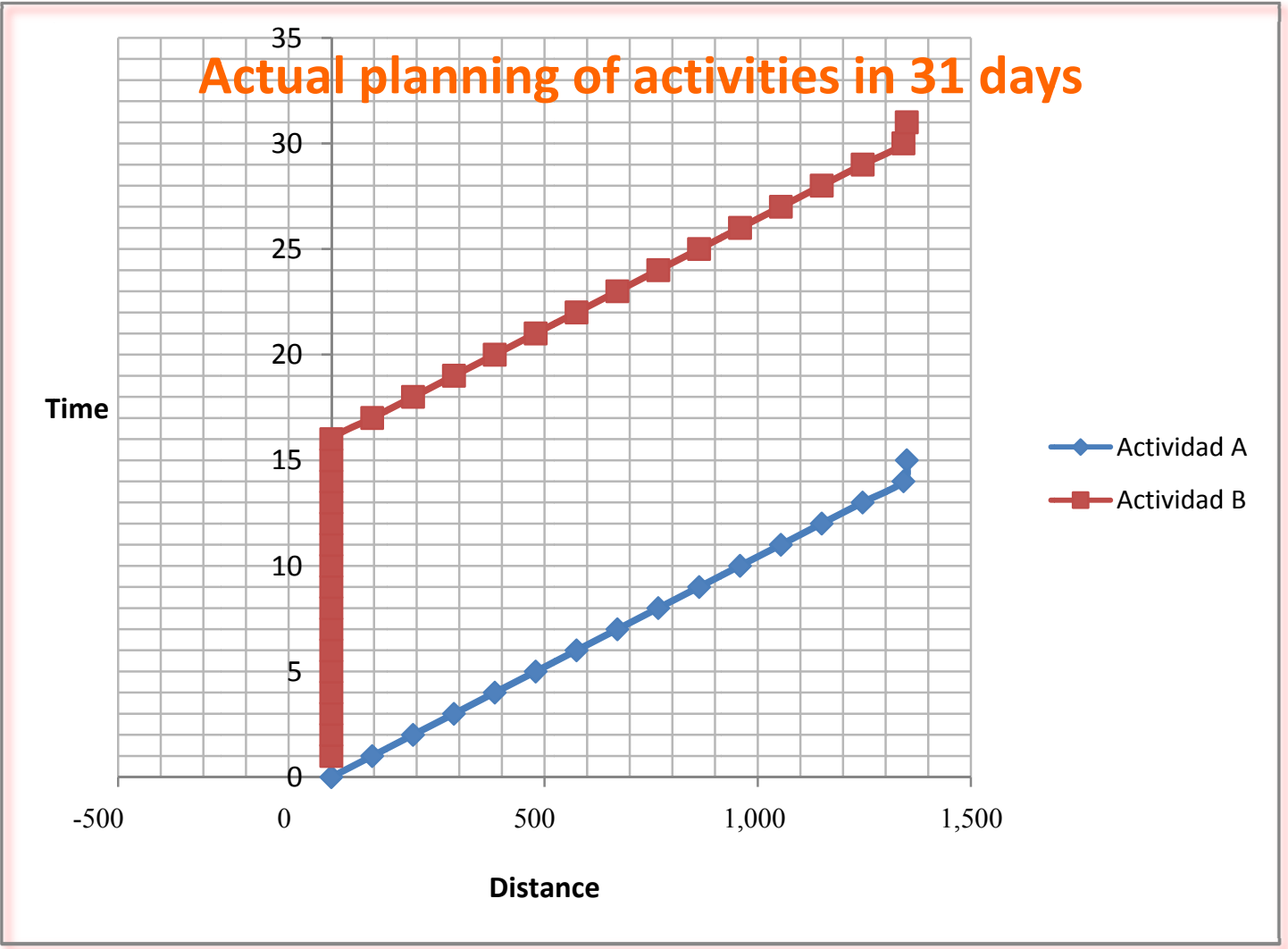

Fig. 1 Duration of the project according to planning in work.

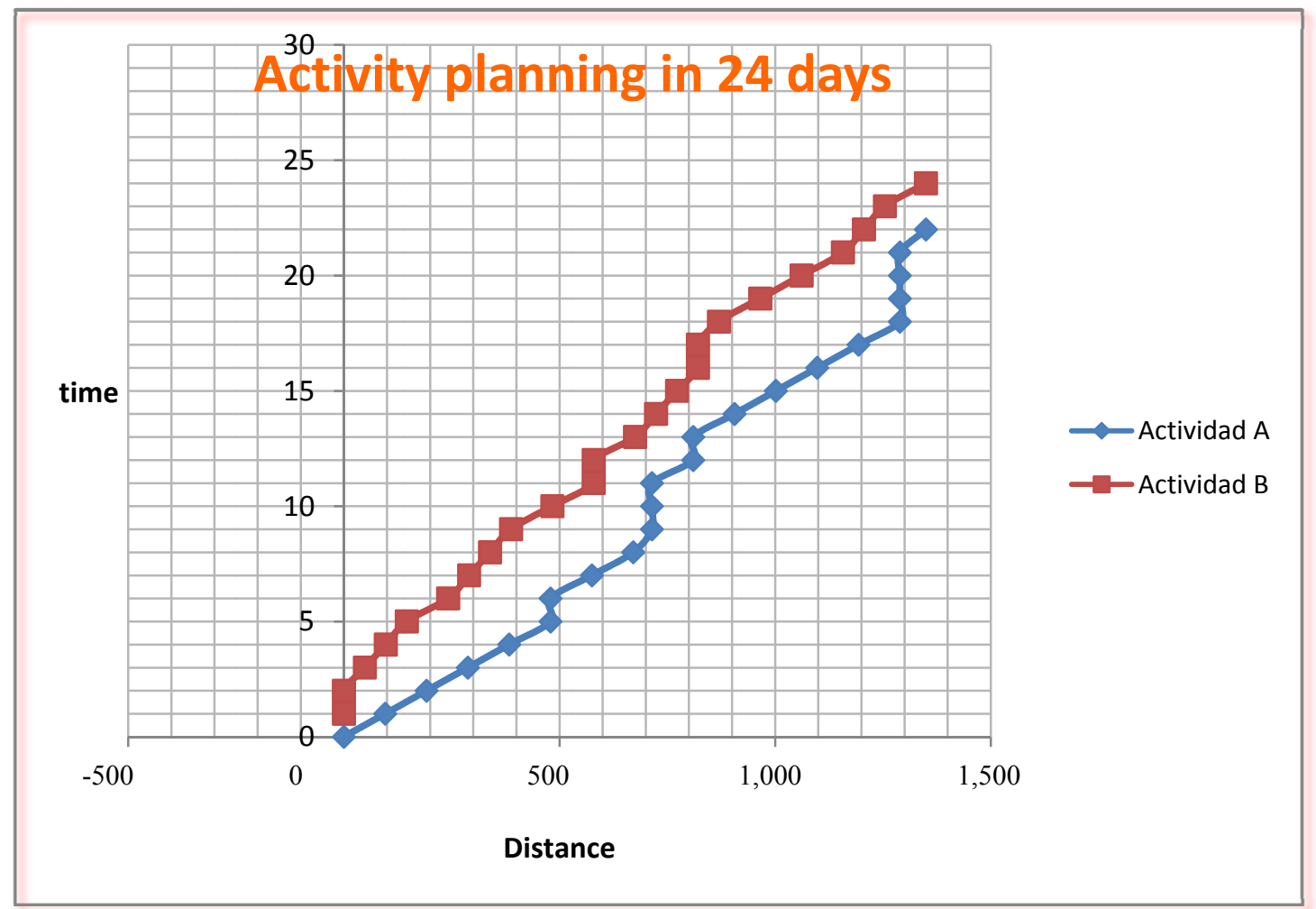

Fig. 2 Optimum of the project. 
we can have with our resources.

\subsection{Restrictions of Work}

In Table 6, we show the restriction appointed of resource. We have to put girders, minimum one day (LT) with daily performance of 95.85 linear meters (LD) for after position plates.

\subsection{Duration of the Project According to Planning in Work}

Fig. 1 shows the planning of work that the construction company pretended run. They finish Activity A and after start Activity B.

\subsection{Optimum of the Project}

Fig. 2 shows the optimal plan using our model of linear programming.

\section{Conclusions}

A study of a real problem is located in limited resources point. We show a mathematical model of mixed integer linear programming. Comparing the actual execution time of the work with the time provided by the mathematical formulation, it is proposed a model of linear programming to solve the problems of the current work of Granadilla and compared it to the actual work done by the company. We concluded that while the company tried to complete the work, developing two consecutive activities at the same time, during 31 days, the problem of combinatorial optimization prepared would develop it in only 24 days, which would suppose a reduction of the term of execution in one week. And we can observe that in the majority of mono-objective consulted works, the aim is to reduce the project time.

\section{References}

[1] J.W. Huang, X.X. Wang, R. Chen, Genetic algorithms for optimization of resource allocation in large scale construction project, Journal of Computers 5 (12) (2010) 1924-1926.

[2] H. Li, N. Chan, H.L. Guo, W. Lu, M. Skitmore, Optimizing construction planning schedules by virtual prototyping enabled resource analysis, Automation in Construction 18 (7) (2009) 912-918.
[3] C. Srisuwanrat, F.G. Ioannou, O. Tsimhoni, Simulation and optimization for construction repetitive projects using promodel and simrunner, in: Proceedings of the 2008 Winter Simulation Conference, Washington D.C., USA, 2008.

[4] Z.Y. Chen, Z.D. Du, H. Zhou, Research on the unlimited resource leveling optimization with PSO (particle swarm optimization), China Civil Engineering Journal 40 (2) (2007) 93-96.

[5] J. He, Z.P. Wan, G.C. Tang, Construction Project Scheduling Problem with Uncertain Resource Constraints, Optimization [Online], http://www.optimization-online.org/DB_HTML/2004/06/ 884.html (accessed Jan. 1, 2014).

[6] L.Y. Zhang, G. Luo, N. Li, Genetic algorithms in resource optimization of construction project, Journal of Tianjin University (Science and Technology), Feb. 2001.

[7] S.S. Leu, C.H. Yang, J.C. Huang, Resource leveling in construction by genetic algorithm-based optimization and its decision support system application, Automation in Construction 10 (1) (2000) 27-41.

[8] L. Gunnar, Integrating efficient resource optimization and linear schedule analysis with singularity functions, Journal of Construction Engineering and Management 137 (1) (2011) 45-55.

[9] K. Amr, E.R. Khaled, E.A. Omar, Optimization research: Enhancing the robustness of large-scale multi-objective optimization in construction, Journal of Construction Engineering and Management 13 (1) (2010) 17-25.

[10] Q.F. Li, J.X. Guo, M.X. Yi, Optimization study on resource equilibrium with fixed time limit for a project based on SPSO algorithm, in: International Symposium on Intelligent Information Technology Application Workshops, Washington, DC, USA, 2008.

[11] H. Fathi, A. Afshar, Multiple resource constraint time-cost-resource optimization using genetic algorithm, in: First International Conference on Construction in Developing Countries, Karachi, Pakistan, Aug. 4-5, 2008.

[12] A.A. Kandil, Multi-objective optimization for large-scale highway construction projects, Ph.D. Thesis, University of Illinois, 2006.

[13] T.M. Cheng, C.W. Feng, Y.L. Chen, A hybrid mechanism for optimizing construction simulation models, Automation in Construction 14 (2005) 85-98.

[14] O.O. Ugwu, J.H.M. Tah, Development and application of a hybrid genetic algorithm for resource optimization and management, Engineering, Construction and Architectural Management 9 (4) (2002) 304-317.

[15] K. El-Rayes, O. Moselhi, Optimizing resource utilization for repetitive construction projects, Journal of Construction Engineering and Management 127 (1) (2001) 18-27.

[16] H. Ching, J. Chang, C. Huang, I. Yang, Resource-constrained scheduling for continuous repetitive projects withsteels-Based production units, Automation in Construction 18 (7) (2009) 942-949. 